\title{
Morganella sulcatostoma (Agaricales, Basidiomycota): Primer hallazgo en Argentina
}

\author{
LEANDRO PAPINUTTI ${ }^{1}$
}

\begin{abstract}
Summary: Morganella sulcatostoma (Agaricales, Basidiomycota), first report for Argentina.
The occurrence of M. sulcatostoma, recently described from Brazil, in Argentina is reported for the first time. Data on macro- and micromorphology of gasterothecia are provided.
\end{abstract}

Key words: gasteromicetes, paracapillitium, taxonomy.

Resumen: Se reporta por primera vez en Argentina, la presencia de $M$. sulcatostoma, especie solo conocida en Brasil. Se aportan datos macro- y micromorfológicosde los gasterotecios.

Palabras clave: gasteromycetes, paracapilicio, taxonomía.

\section{INTRODUCCIÓN}

El género Morganella Zeller tiene una amplia distribución geográfica y de hábitat, diferentes especies fueron halladas en regiones tropicales y templadas (Ponce de León, 1971; Suárez \& Wright, 1996; Gube \& Piepenbring, 2009), aunque no fue reportada ninguna especie creciendo en el desierto. Tal cual como fue notado por Kreisel y Dring (1967), el género posee especies mayoritariamente lignícolas, rara vez en suelo arenoso (Barbosa et al., 2011) o terrícolas, sin embargo, a Morganella fuliginea es común hallarla creciendo sobre materia orgánica en el suelo(Suárez \& Wright, 1996; Wright \& Wright, 2005).

El género se caracteriza por sus gasterotecios globosos a veces algo aplanados, pequeños de hasta $3 \mathrm{~cm}$ diam, peridio formado por dos capas, la más externa o exoperidio, que en algunas especies se cae, cuando el basidioma envejece, dejando al descubierto la capa interna o endoperidio, el cual se abre por un poro apical. El exoperidio puede ser furfuráceo, granular, afelpado, verrugoso o espinoso carácter de valor taxonómico a nivel específico. La

\footnotetext{
1 Laboratorio de Micología Experimental, Facultad de Ciencias Exactas y Naturales, Universidad de Buenos Aires. Correo electrónico: leandru@bg.fcen.uba.ar
}

gleba es pulverulenta y está constituida por esporas y paracapilicio cuyas hebras están unidas por membranas glebales; el género carece de capilicio verdadero.

El concepto genérico seguido en este trabajo corresponde a la delimitación propuesta por Kreisel y Dring (1967) basada en la micromorfología quedando Lycoperdon, un género casi indistinguible por la macromorfología de Morganella, bien delimitado.

Datos moleculares de secuencias de ADN obtenidos en los últimos años han comenzado a modificar gradualmente los agrupamientos. Larsson y Jeppson (2008) construyeron la filogenia molecular y en base a este estudio sugieren que Apioperdon, Bovistella, Morganella, Utraria y Vascellum debieran ser subdivisiones de un género más abarcativo de Lycoperdon. Sin embargo en este estudio no han incluido especies del Hemisferio Sur, quedando por lo tanto su filogenia sin resolver. Es por esto, que en este trabajo se mantienen los diferentes géneros como tales.

La revisión de Morganella hecha por Suárez \& Wright (1996) para Sudamérica da cuenta de tres especies, M. costaricencis M.I. Morales, $M$. fuliginea y $M$. velutina (Berk. ex Massee) Kreisel \& Dring, sin embargo en estos últimos años se describieron nuevas especies y registraron 
nuevas citas, especialmente de Brasil (Trierveiler \& Baseia 2009; Barbosa et al., 2011; Alfredo et al., 2012). Trabajos más recientes de prospecciones de hongos gasteroides en Argentina no reportaron la ocurrencia de ninguna especie de este género (Dios et al., 2011; Kuhar et al., 2012; Hernández-Caffot et al., 2013).

Este trabajo reporta la primer cita para Argentina de M. sulcatostoma, una especie recientemente descrita en Brasil (Alves \& Cortez, 2013), cuyo carácter diagnóstico más sobresaliente es su peristoma surcado.

\section{Materiales y Métodos}

El estudio y las descripciones se realizaron en base al material colectado y seco, siguiendo la metodología y terminología propuesta por Kreisel\&Dring (1967). La colección fue secada en estufa a $40^{\circ} \mathrm{C}$ durante 24 $\mathrm{h}$, luego congelada a $-20^{\circ} \mathrm{C}$ y guardada en recipiente hermético y depositada en el herbario de la Facultad de Ciencias Exactas y Naturales de la Universidad de Buenos Aires (BAFC).

Las características macroscópicas fueron observadas a ojo desnudo y bajo binocular estereoscópico.

Los caracteres microscópicos se observaron bajo microscopio óptico luego de teñir con floxina B (Sigma-Aldrich, St. Louis, MO) al 5\%. Para la observación de las membranas glebales se siguió la metodología propuesta por Kreisel \& Dring (1967). El rango de medidas de las esporas es resultado de las mediciones de al menos 20 esporas por preparado, se midieron las esporas de 3 basidiomas diferentes.

\section{Resultados}

Morganella sulcatostoma C.R. Alves \& Cortez, Nova Hedwigia 96: 409. (2013). Figs. 1 y 2.

Material estudiado. ARGENTINA. Prov. Misiones: Dpto. Aristóbulo del Valle, Valle del Cuña Pirú, $27^{\circ} 05^{`} 28^{\prime \prime} \mathrm{S}$; $54^{\circ} 55^{`} 32^{\prime \prime}$ O., 15-III2009, L. Papinutti 52327 (BAFC).

\section{Macromorfología}

Gasterotecios pequeños, epígeos, $0,8-1,8 \mathrm{~cm}$ alto Í 0,8-1,6 cm diam, globosos o piriformes (Fig.
1A); peridio doble, exoperidio compuesto de cortas espinas de hasta $0,9 \mathrm{~mm}$, blanco, desprendiéndose en placas del endoperidio a medida que madura; endoperidio pardo claro, papiráceo, se abre por un poro apical rodeado de un peristoma plegado (Fig. 1B); subgleba y pseudocolumela ausentes; gleba pulverulenta, marrón claro; rizoides presentes, 2-6, delgados de hasta $0,1 \mathrm{~mm}$ diam, blancos, persistentes, con incrustaciones de material particulado del suelo.

\section{Micromorfología}

Espinas del exoperidio pseudoparenquimáticas, células rectangulares a elípticas (Fig. 1A), dispuestas en cadenas, pared delgada, hialinas; endoperidio (compuesto por hifas cuya pared está pigmentada (Fig. 2B), pardo amarillentas, 2-4 $\mu \mathrm{m}$ diam, paredes gruesas $(<1 \mu \mathrm{m})$, sin incrustaciones de ningún tipo; micoesclereidas (Fig. 2C) tapizando

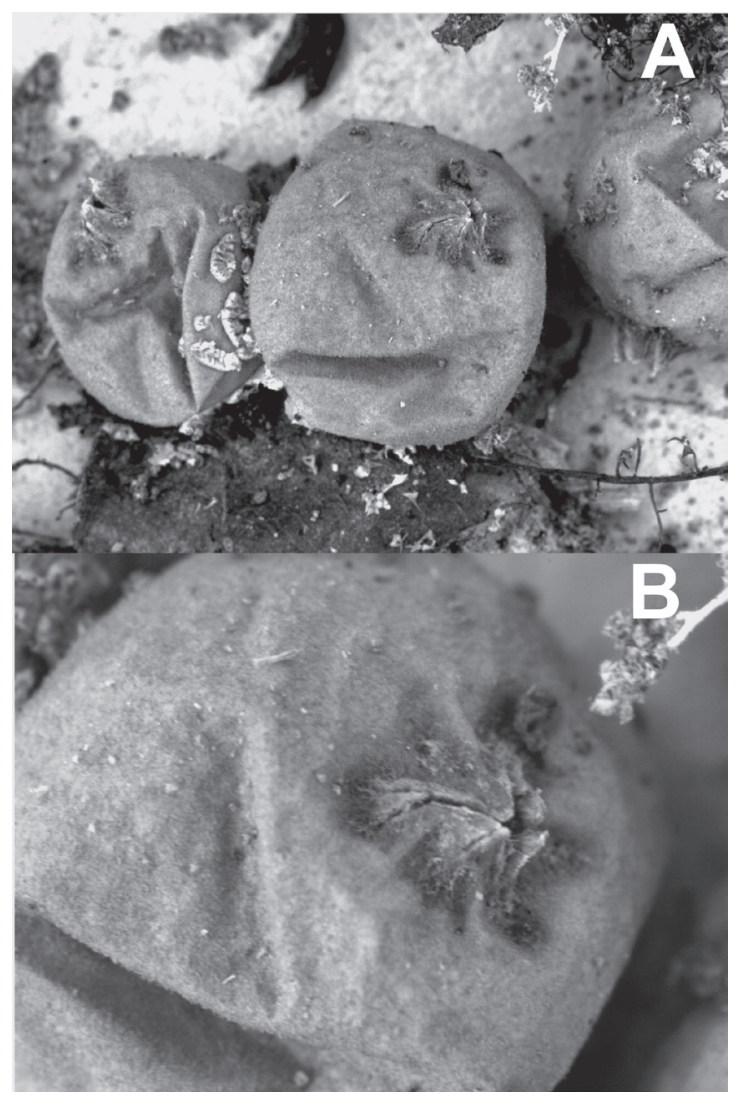

Fig. 1. Morganella sulcatostoma BAFC 52327. A: Aspecto general de los gasterotecios. B: Detalle del peristoma plegado. 


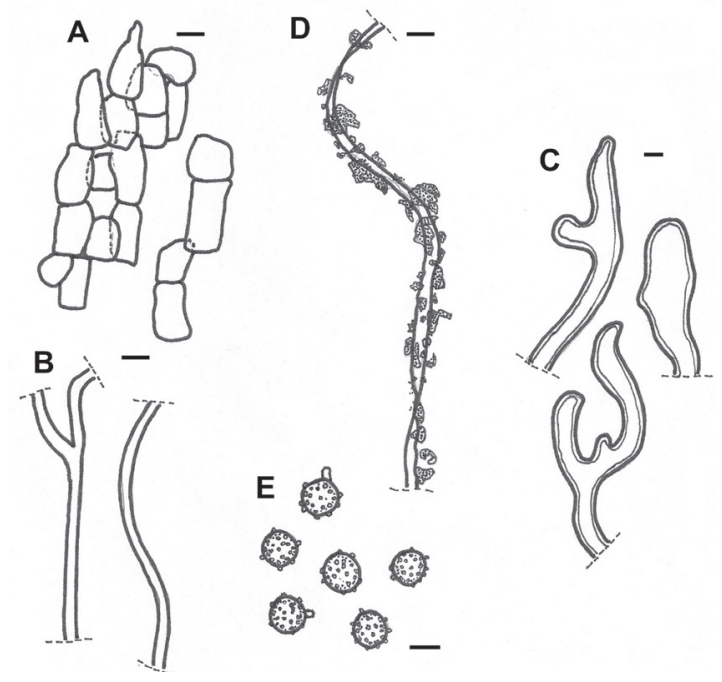

Fig. 2. Morganella sulcatostoma: micromorfología. A: Células pseudoparenquimáticas del exoperidio. B: Hifas del endoperidio. C: Micoesclereidas del endoperidio. Hifas del endoperidio. D: Paracapilicio con incrustaciones. E: Esporas. La barra de escala represena en $\mathrm{A}=15 \mu \mathrm{m}$; $\mathrm{y}$ en $\mathrm{B}, \mathrm{C}, \mathrm{D}$ y $\mathrm{E}=5 \mu \mathrm{m}$.

la superficie del endoperidio, $20-100 \mu \mathrm{m}$ alto $\mathrm{x}$ 8-13 $\mu \mathrm{m}$ diam, pared gruesa $1 \mu \mathrm{m}$, cortamente ramificadas en los extremos; eucapilicio ausente; paracapilicio (Fig. 2D) abundante, 3-4 $\mu \mathrm{m}$ diam, paredes delgadas, hialinas con membranas glebales y otros elementos amorfos incrustados; esporas globosas, 5,0-5,8 $\mu \mathrm{m}$ diam, ornamentadas con conspicuas verrugas, pedicelo corto $<1 \mu \mathrm{m}$ (Fig. 2E).

\section{Hábito y hábitat}

Gregarios, en suelo sobre mantillo de hojas y pequeñas ramas.

\section{Observaciones}

La colección tipo de Brasil fue hallada en un ambiente muy similar (Alves \& Cortez, 2013) a aquel donde se encontró la colección que se describe en este trabajo. Dado que no sólo corresponde al mismo dominio fitogeográfico, sino que también fueron encontrados creciendo sobre hojas caídas aunque de especies diferentes de plantas, la colección de Brasil fue encontrada sobre hojas de Syagrus romanzoffiana mientras que la colección argentina se halló creciendo sobre hojarasca de diferentes especies de plantas. Si bien la colección tipo da cuenta de gasterotecios de hasta $1 \mathrm{~cm}$ de alto, los especímenes de la colección de Misiones son más grandes, llegando a $1,8 \mathrm{~cm}$ al igual que las esporas que son algo más grandes $(5,0-5,8 \mu \mathrm{m})$ que las de la colección tipo. Los demás caracteres tanto macromorfológicos como micromorfológicos de la colección argentina son idénticos y coinciden con el rango de medidas de la colección tipo.

El género Morganella fue escasamente estudiado en Argentina siendo el trabajo de Suárez y Wright (1996) el único que lo trató de manera más abarcativa y donde solo se describen tres especies. Dado que se trata de un género que se encuentra con mayor frecuencia en ambientes de bosque húmedo y que en Argentina estos fueron poco explorados, particularmente la región de selva Valdiviana, podemos considerar que el número de especies puede ser considerablemente mayor que las descriptas hasta el momento. En este trabajo se reporta la primera cita de M. sulcatostoma para nuestro país ampliando así su área de distribución la cual hasta el momento estaba limitada a Brasil.

\section{Agradecimientos}

Este trabajo fue financiado por CONICET (Proyecto PIP0413) y la Universidad de Buenos Aires (Proyecto UBACYT 0049).

\section{Bibliografía}

ALFREDO, D. S., A. G. LEITE, R. BRAGA-NETO \& I. BASEIA. 2012. Two new Morganella species from the Brazilian Amazon rainforest. Mycosphere 3: 6671.

ALVES, C. R. \& V. G. CORTEZ. 2013. Morganellasulcatostomasp. nov. (Agaricales, Basidiomycota) from Paraná State, Brazil. Nova Hedwigia 96: 409-417.

BARBOSA, M., M. SILVA, R. CRUZ, F. CALONGE \& I. BASEIA. 2011. First report of Morganellacompacta (Agaricales, Lycoperdaceae) from South America. Mycotaxon 116: 381-386.

DIOS, M.M., E. ALBERTÓ \& G. MORENO. 2011. Catálogo de hongos gasteroides (Basidiomycota) de Catamarca, Argentina. Bol. Soc. Argent. Bot. 46: 5-11.

GUBE, M. \& M. PIEPENBRING. 2009. Preliminary annotated checklist of Gasteromycetes in Panama. Nova Hedwigia 89: 519-543. 
HERNÁNDEZ-CAFFOT, M.L., G. ROBLEDO \& L. S. DOMÍNGUEZ. 2013. Gasteroidmycobiota (Basidiomycota) from Polylepisaustralis woodlands of central Argentina. Mycotaxon 123: 491. Disponible en: http://www.mycotaxon.com/ resources/checklists/Hernandez-v123-checklist.pdf [Acceso: Junio 2013].

KREISEL, H. \& D. M. DRING. 1967. An emendation of the genus Morganella Zeller (Lycoperdaceae). Fedes Repert. 74: 109-122.

KUHAR, F., V. CASTIGLIA, J. ZAMORA\& L. PAPINUTTI. 2012. New records and notes on gasteroid fungi of arid regions in Argentina. Sydowia 64: 233-244.

LARSSON, E. \&M. JEPPSON. 2008. Phylogenetic relationships among species and genera of Lycoperdaceae based on ITS and LSU sequence data from north European taxa. Mycol. Res. 112: 4-22.
PONCE DE LEÓN, P. 1971. Revision of the genus Morganella (Lycoperdaceae). Fieldiana, Bot. 34: 27-44.

SUÁREZ, V. \& J. WRIGHT. 1996. South American Gasteromycetes V: The genus Morganella. Mycologia 88: 655-661.

TRIERVEILER-PEREIRA， L. \&I. G. BASEIA. 2009. A checklist of the Brazilian gasteroid fungi (Basidiomycota). Mycotaxon 108: 441-444.

WRIGHT, J. E. \& A. WRIGHT. 2005. Checklist of the mycobiota of Iguazú National Park (Misiones, Argentina). Bol. Soc. Argent. Bot. 40: 23-44.

Recibido el 26 de agosto de 2013, aceptado el 19 de septiembre de 2013. 